\title{
X-ray diffraction study of the composition and strain fields in buried SiGe islands
}

\author{
N. Hrauda ${ }^{1, a}$, J.J. Zhang ${ }^{1}$, M. Stoffel ${ }^{2}$, J. Stangl ${ }^{1}$, G. Bauer ${ }^{1}$, A. Rehman-Khan ${ }^{1}$, V. Holý ${ }^{3}$, \\ O.G. Schmidt ${ }^{2}$, V. Jovanovic ${ }^{4}$, and L.K. Nanver ${ }^{4}$ \\ 1 Institute for Solid State and Semiconductor Physics, Johannes Kepler University of Linz, \\ Altenbergerstrasse 69, 4040 Linz, Austria \\ 2 IWF Dresden, Helmholtzstrasse 20, 01069 Dresden, Germany \\ 3 Faculty of Mathematics and Physics, Charles University Prague, Ke Karlovu 5, Praha, \\ 12116, Czech Republic \\ 4 TU Delft, Stevinweg 1, 2628, The Netherlands
}

\begin{abstract}
We report on studies of strain and composition of two-dimensionally ordered SiGe islands grown by molecular beam epitaxy using high resolution $\mathrm{x}$-ray diffraction. To ensure a small size distribution of the islands, pit-patterned $4^{\prime \prime}$ (001) Si wafers were used as substrates. The $\mathrm{Si}$ wafers were patterned by optical lithography and reactive ion etching. The pits for island growth are ordered in regular 2D arrays with periods ranging from 500 to $1000 \mathrm{~nm}$ along two orthogonal $\langle 110\rangle$ directions. After the growth of a Si buffer layer, 5 to 9 monolayers of Ge are deposited, leading to the formation of islands with either dome- or barn shape, depending on the number of monolayers deposited. The $\mathrm{Si}$ capping of the islands is performed at low temperatures $\left(300^{\circ} \mathrm{C}\right)$ to avoid intermixing and thus strain relaxation. Information on the surface morphology obtained by atomic force microscopy (AFM) was used to set up models for three-dimensional Finite Element Method (FEM) simulations of the islands including the patterned Si substrate. In the model, special attention was given to the non uniform distribution of the Ge content within the islands. The FEM results served as an input for calculating the diffracted x-ray intensities using kinematical scattering theory. Reciprocal space maps around the vicinity of symmetric (004) and asymmetric (113) and (224) Bragg peaks were recorded in coplanar geometry. Simulating different germanium gradients leads to altered scattered intensity distribution and consequently information on this quantity is obtained for both dome- and barn-shaped islands as well as on the strain fields.
\end{abstract}

\section{Introduction}

SiGe islands on (001) Si constitute a model system for the investigation of Stranski-Krastanow self-organized growth [1]. The lattice mismatch of $4.2 \%$ between Ge and Si leads to island formation for Ge deposition exceeding about 4 monolayers. For any application such islands have to be capped with Si. The Si capping layer will be tensile strained, where the amount of strain depends on the size, shape, Ge content and relaxation state of the islands. Even if pure Ge is deposited, due to the elevated growth temperatures of about $700{ }^{\circ} \mathrm{C}$, interdiffusion and segregation processes change the composition and thus the island's strain state. Due to the statistical nature of the growth process if deposited on planar Si substrates, such islands usually exhibit a rather broad size distribution. By growing Ge islands on two-dimensionally pit-patterned Si

\footnotetext{
a e-mail: nina.hrauda@jku.at
} 


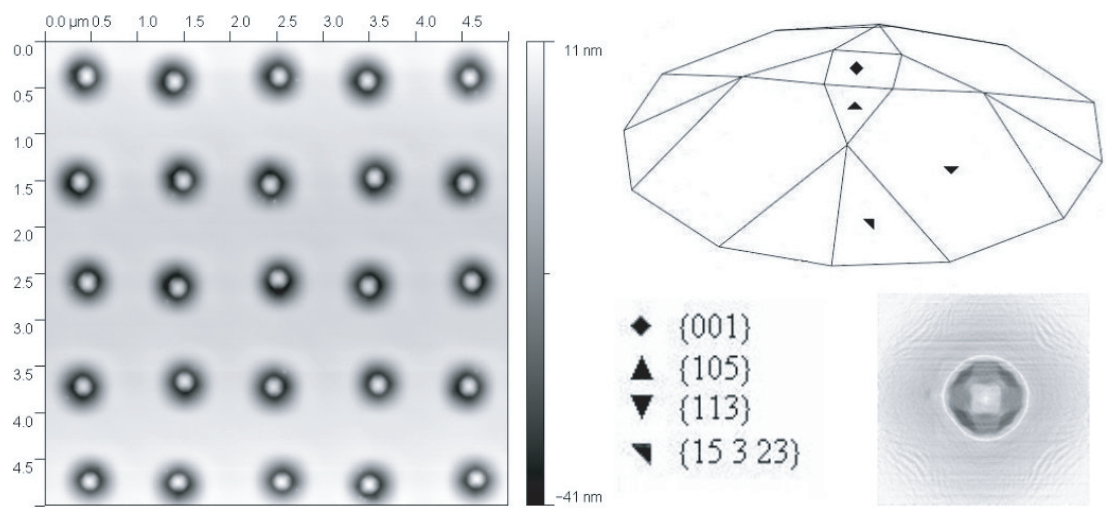

Fig. 1. The left part shows an AFM image of dome-shaped ordered islands (period of $1000 \mathrm{~nm}$ ). The upper right part shows a schematic sketch of a such an island with labelled facets. The small inset below shows a more detailed image of a single dome to visualise single facets.

substrates, the island size distribution is much narrower [2-5]. Furthermore such islands can be addressed individually if necessary, e.g. for the fabrication of electronic devices [6,7]. In this paper we report on systematic studies of strain and composition of two-dimensional arrays of dome- and barn-shaped SiGe islands with periods between 500 and $1000 \mathrm{~nm}$, which were grown by molecular beam epitaxy $(\mathrm{MBE})$. For their structural characterization atomic force microscopy (AFM) and high resolution x-ray diffraction (XRD) using synchrotron sources were used. Information on the Ge content of the islands and their strain state was obtained from a comparison of calculated x-ray diffraction data based on kinematical theory $[1,9]$ with the experimental reciprocal space maps. As input for these simulations the displacement data of three-dimensional Finite Element Method (FEM) calculations were used [8].

\section{Experimental - sample growth and X-ray diffraction}

Periodically patterned Si substrates were produced by optical lithography and reactive ion etching with pits oriented along two orthogonal $\langle 110\rangle$ directions and depths of about $50 \mathrm{~nm}$. For the deposition of a Si buffer layer with thicknesses in between 18 and $36 \mathrm{~nm}$, the growth temperature was ramped from $360^{\circ} \mathrm{C}$ to $500^{\circ} \mathrm{C}$. Then several monolayers (ML) of Ge were deposited at a temperature of $700^{\circ} \mathrm{C}$. For more details on the MBE growth see [5]. Whereas the deposition of 5 to $6 \mathrm{ML}$ Ge results in dome-shaped islands, depositing more than $8 \mathrm{ML}$ results in barns. Previous transmission electron microscopy investigations have shown that islands grown under such conditions do not exhibit dislocation formation, actually for islands grown in pits the formation of dislocations is delayed as compared to islands grown on flat substrates, see [10]. Subsequently the islands were capped with $50 \mathrm{~nm} \mathrm{Si}$ at low temperature $\left(300^{\circ}\right)$ to maintain the shape of the dots and avoid intermixing. AFM measurements before and after Si capping prove that for these conditions the shape and facets of the capped islands are preserved. In Fig. 1 AFM data of uncapped domes grown on a wafer with a pit period of $1000 \mathrm{~nm}$ are shown, together with a schematic sketch of the dome shaped island with facet labels and a corresponding high resolution AFM image of a single island. The barn-shaped islands, characterised by additional facets and a higher aspect ratio, will be treated in a later section of this paper (see Fig. 4). High resolution coplanar x-ray diffraction data of such samples were recorded at the beamline BW2, Hasylab, Hamburg and at ID10B, ESRF, Grenoble. Reciprocal space maps around the (004) and (113) reciprocal lattice points were recorded for a wavelength of $1.7712 \AA$ at Hasylab. For the data collection a CCD (charge coupled device) camera was used. By performing 1:2 relative scans around a Bragg peak (which means that relative to the aligned peak position, the range for the detector-angle $\theta$ is twice as large as the incident angle $\omega) 3 \mathrm{D}$ data were collected in a single scan. The second series of samples was measured at the beamline ID10B using a wavelength of $1.5498 \AA$. Scattered intensities were recorded around the 


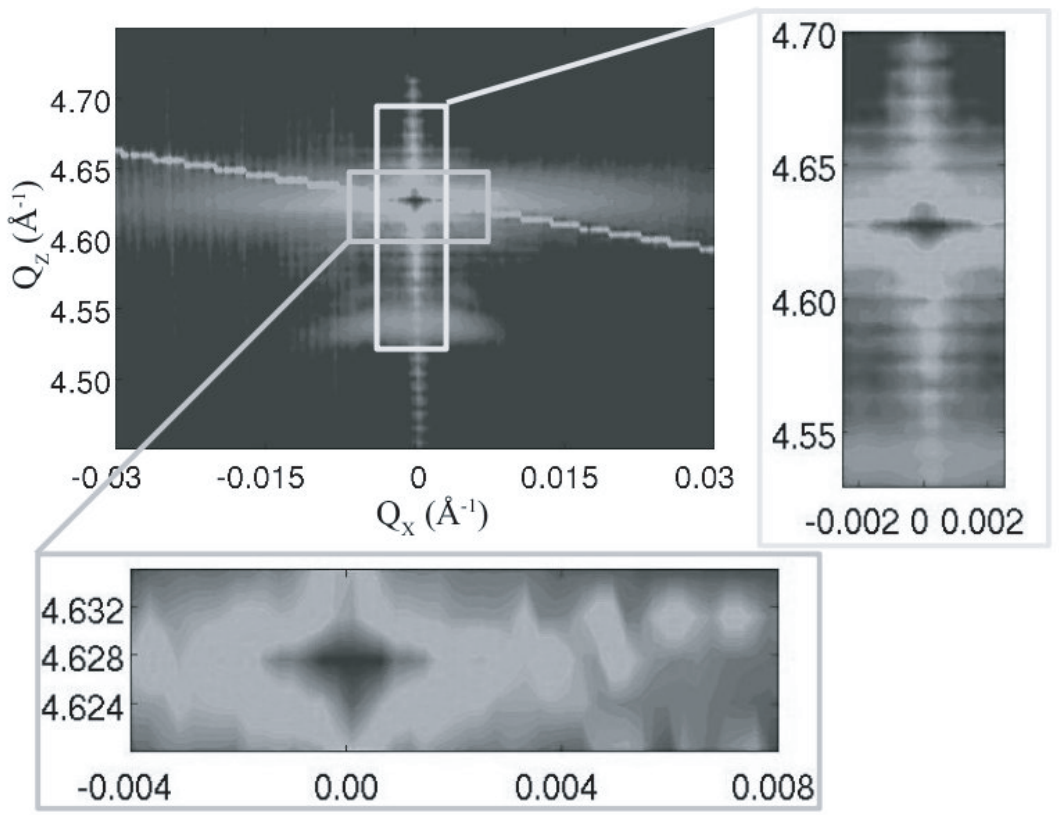

Fig. 2. This figure shows an example for features that can be seen on a symmetrical reciprocal spacemap, here the (004) Bragg peak. This measurement was performed on capped islands, thus the thickness of the Si capping layer $(53 \mathrm{~nm}$ ) leads to vertical intensity oscillations (shown in the right inset). The lateral period can be determined from the intensity osscillations along $\mathrm{Q}_{x}$ displayed in the lower inset (in this example about $535 \mathrm{~nm}$ ). The rather faint signal below the sharp Si peak in the main part of the figure originates from the SiGe island signal.

(004) and (224) reciprocal lattice points with a position sensitive detector. Figure 2 shows as an example a (004) map of a sample with capped dome-shaped islands. Along the truncation rod corresponding to the [001] surface normal, intensity fringes due to the $53 \mathrm{~nm}$ thick capping layer are observed, which accompany the main $\mathrm{Si}$ diffraction spot. In the inset below lateral satellites are shown which are caused by the periodicity of the pit pattern of about $535 \mathrm{~nm}$ along the [110] direction. The diffraction signal which originates from the islands is situated at lower $Q_{z}$ values, below the Si peak.

To obtain detailed information on the variation of the island's Ge content and their strain state we use model assumptions on these parameters, utilizing information on the island sizes and their shapes from AFM data. Based on these island models, finite element simulations yield displacements which are used as input parameters for the calculation of the diffracted intensities.

\section{Finite element simulations}

For the correct description of capped islands in pits, apart from information on the islands, data on the pit shape as well as on the Si capping layer are needed, these are obtained from AFM measurements. Due to the rather large period of the pattern $(500-1000 \mathrm{~nm})$ for the 3D simulations a Si-substrate block with a single island in a pit was considered. After the growth of the Si buffer layer, the pits have the shape of inverted truncated pyramids with $\left[\begin{array}{lll}1 & 1 & 10\end{array}\right]$ side facets. In the FEM model, the islands, either dome or barn shaped, were considered with all their facets, and the shape of the Si capping layer was exactly taken into account, including the trenches surrounding the capped islands [11]. For the islands also the part which fills the inverted pyramid of the pits was considered. The Ge distribution was defined by gradients with increasing Ge towards the island apex in $z$ direction for 1D-gradient models (uncapped domes and barns) and additional gradients with the germanium content decreasing towards the rim 

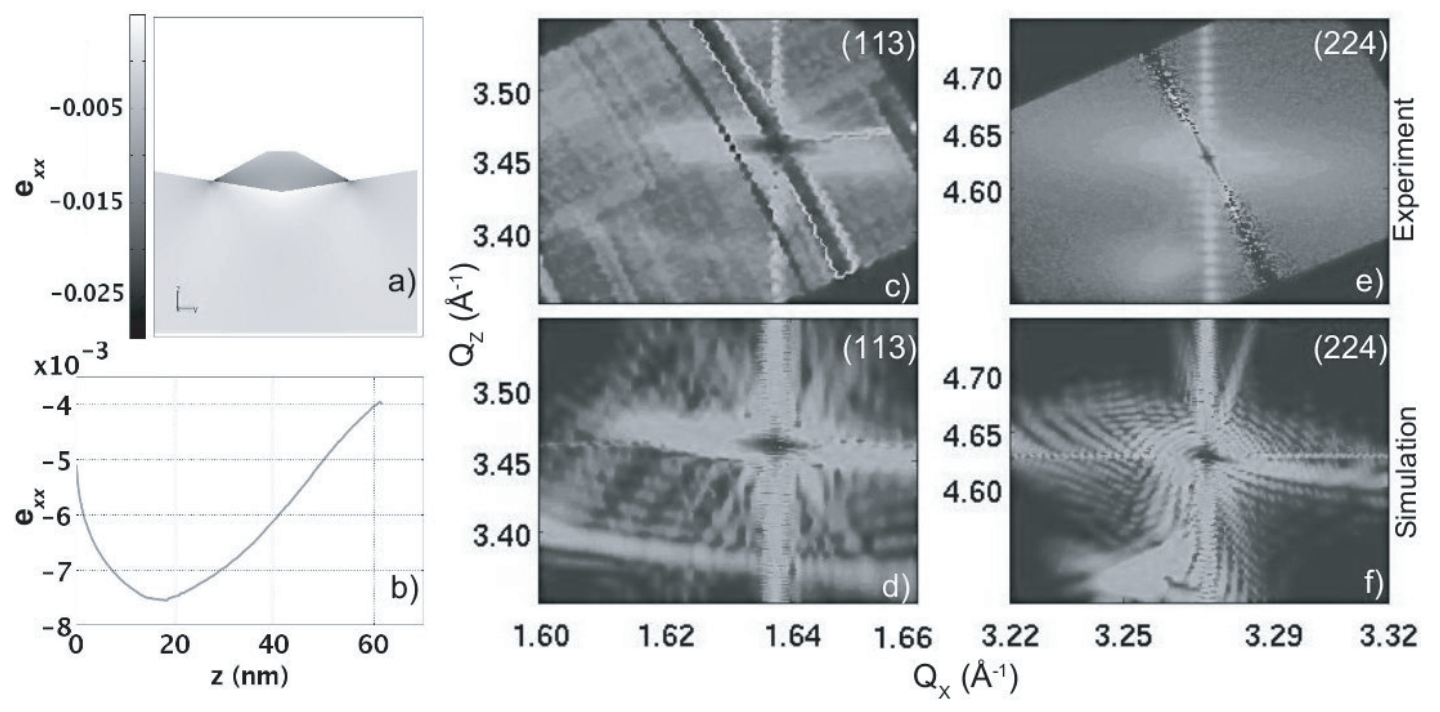

Fig. 3. In the left part of this figure, results of a FEM simulation for an uncapped dome shaped island is shown with a 2D strain plot (Fig. 3(a)) and a vertical line plot of the strain $\mathrm{e}_{x x}$ through the middle of the island (panel b) revealing the relaxation of compressive strain in the island towards the island apex. In the middle and right sections, a comparison of experimental and simulated x-ray data is displayed, with the upper parts showing the measurements and the lower insets the respective simulations derived from FEM models of uncapped (panel (c) and (d)) and capped (panel (e) and (f)) islands.
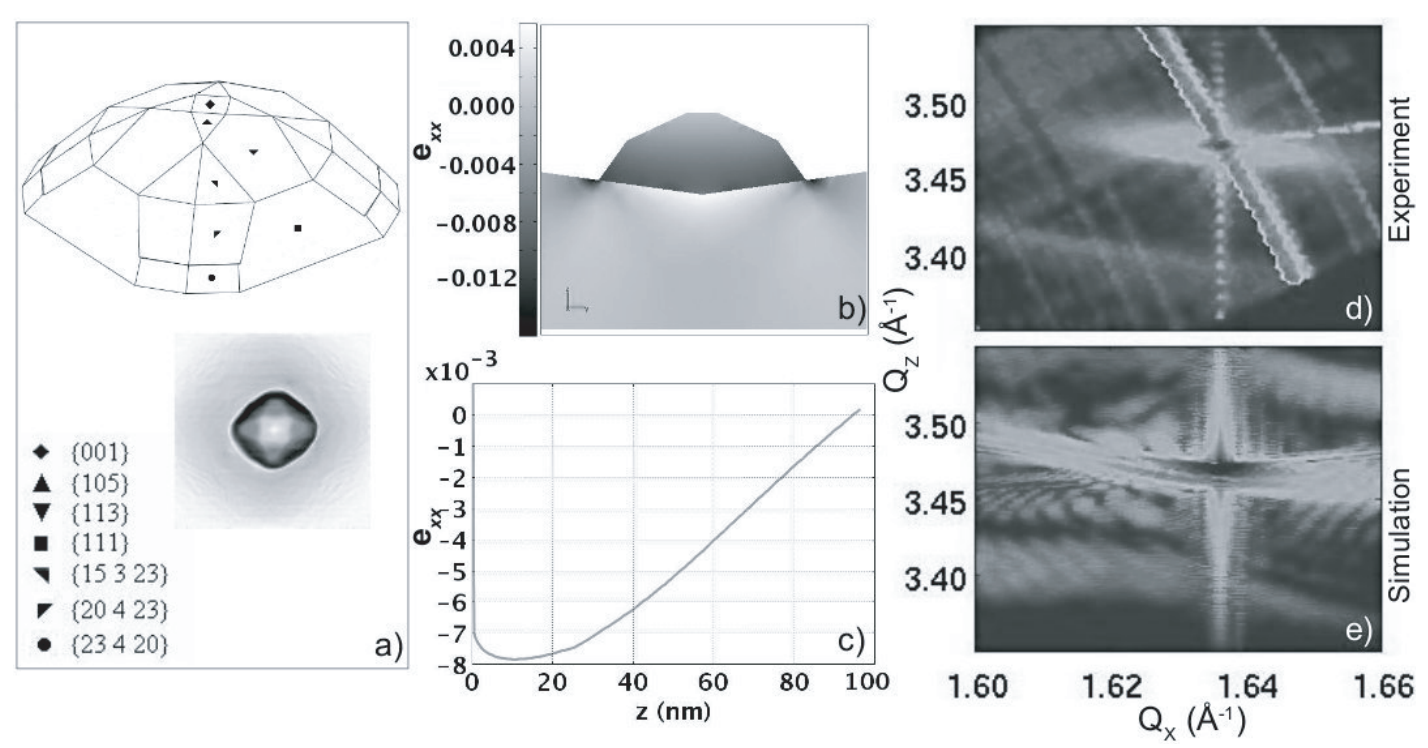

Fig. 4. Schematic sketch of a barn-shaped island with facet labels (a), the small image displays again a detailed AFM measurement of a real barn shaped island. Panels (b) and (c) show plots of the strain component $\mathrm{e}_{x x}$ comparable to Fig. 3, whereas the vertical line plot here shows the relaxed state at the apex of a barn. In Fig. 4(d) x-ray data around the asymmetric (113) Bragg peak obtained from a sample with barn shaped islands are shown with the according x-ray simulation (panel (e)) derived from the FEM model shown in the middle part.

of the islands for 3D distributions (capped domes). With the data extracted from the 3D finite elements simulations the 2D intensity distribution in the reciprocal space maps was calculated and subsequently compared to the measured data. Examples for the analysis of diffraction data from uncapped dome shaped islands are shown in Fig. 3(c) (Experiment) and 3(d) (Simulation), 
the respective data for capped islands are shown in Fig. 3(e) and 3(f). We compare the measured reciprocal space map around an asymmetrical (113) reciprocal lattice point with simulations, for which the 3D island model assumptions across a section corresponding to a (110) plane are shown for the strain tensor component $\mathrm{e}_{x x}$ (2D plot in Fig. 3(a) and corresponding plot along a vertical line through the middle of the island in Fig. 3(b)). The diameter of the island is $240 \mathrm{~nm}$, its height $45 \mathrm{~nm}$, i.e. the corresponding aspect ratio is 0.19 . The maximum Ge content is $45 \%$, the maximum value of $\mathrm{e}_{x x}$, within the island is about -0.0075 (defined with respect to the corresponding relaxed $\mathrm{SiGe}$ alloy). The important result of this simulations with respect to applications is the amount of tensile strain in the Si cap layer. For the $50 \mathrm{~nm}$ thick cap layer, the tensile strain (with respect to bulk $\mathrm{Si}$ ) is approximately constant and yields a value of $0.8 \%$. To achieve an even higher tensile strained Si cap layer, the degree of relaxation within the SiGe island has to be increased which could be achieved by a higher Ge content and/or aspect ratio. The aspect ratio of barn shaped islands is 0.32 , their average Ge content is about $40 \%$. Figure 4 shows a model of the barn with all facets depicted, high resolution AFM data of a single barn in a pit, (113) reciprocal space maps and simulated ones based on the corresponding island model. For this barn model the strain distribution is shown for an island diameter of $240 \mathrm{~nm}$ and a height of $77 \mathrm{~nm}$. As expected, the $\mathrm{e}_{x x}$ values close to the top of the barn are indeed very close to zero, corresponding to nearly complete relaxation. Due to the fact, that a high degree of relaxation within the island itself induces high tensile strains in a silicon capping layer, these first results of samples containing uncapped barns are very promising. Investigations on Si capped barns are currently underway.

\section{Conclusions}

Precise information on the strain fields and Ge content of SiGe islands grown on pit-patterned $\mathrm{Si}$ (001) wafers was obtained from an analysis of high resolution x-ray diffraction data recorded in coplanar geometry. To achieve this goal, AFM data on the size and shape of the islands as well as of the pits were used as an input to three-dimensional finite element simulations. The displacements as obtained from the model served as an input for calculations of the scattered intensities. The Ge content within the island was varied both vertically as well as laterally in the FEM model simulations until a reasonable fit between the calculated and measured x-ray diffraction patterns was achieved. From our measurements and simulations it turns out that in the $50 \mathrm{~nm}$ Si capping layer which covers the dome-shaped Ge islands tensile strain values of up to $0.8 \%$ are feasible. This value is about a factor of three higher than the one which can be realized by capping relaxed SiGe epilayers with Si without introducing dislocations. Consequently, the reported x-ray diffraction investigations prove that the concept of realizing highly strained $\mathrm{Si}$ channels by exploiting the strain state in the Si capping layers on top of periodically aranged islands as suggested by Schmidt and Eberl [6] is superior to concepts based on buried relaxed SiGe epilayers [12].

We thank the staff at BW2 at Hasylab and at ID10B at the ESRF for their support and G. Vastola, A. Marzegalli, F. Montalenti and L. Miglio for discussions on the 3D FEM simulations. This work was supported by the FWF, Vienna (SFB025) and the EC d-DOTFET project (012150-2).

\section{References}

1. J. Stangl, V. Holý, G. Bauer, Rev. Mod. Phys. 76, 725 (2004)

2. Z. Zhong, A. Halilovic, M. Mühlberger, F. Schäffler, G. Bauer, Appl. Phys. Lett. 82, 4779 (2003)

3. Z. Zhong, G. Bauer, Appl. Phys. Lett. 84, 1922 (2004)

4. D. Grützmacher, T. Fromherz, C. Dais, J. Stangl, E. Müller, Y. Ekinci, H.H. Solak, H. Sigg, R.T. Lechner, E. Wintersberger, S. Birner, V. Holý, G. Bauer, Nano Lett. 7, 2447 (2007)

5. J.J. Zhang, M. Stoffel, A. Rastelli, O.G. Schmidt, V. Jovanovic, L.K. Nanver, G. Bauer, Appl. Phys. Lett. 91, 173115 (2007) 
6. O.G. Schmidt, K. Eberl, IEEE Trans. Electron Devices 48, 1175 (2001)

7. K.W. Ang, C.H. Tung, N. Balasubramanian, G.S. Samudra, Y.C. Yeo, IEEE Electron Dev. Lett. 28, 609 (2007)

8. Comsol Multiphysics Program Package

9. see e.g., U. Pietsch, V. Holý, T. Baumbach, High resolution X-ray scattering from thin films and nanostructures (Springer Berlin, Heidelberg, New York, 2004)

10. Z. Zhong, W. Schwinger, F. Schäffler, G. Bauer, G. Vastola, F. Montalenti, L. Miglio, Phys. Rev. Lett. 98, $176102(2007)$

11. G. Vastola, A. Marzegalli, F. Montalenti, L. Miglio, Mater. Sci. Eng. B (2008) (in print)

12. R.A. Donaton, D. Chidambarrao, J. Johnson, P. Chang, Y. Liu, W. Kirklen Henson, J. Holt, X. Li, A. Domenicucci, A. Madan, K. Kim, C. Wann, Tech. Dig. - Int. Electron Devices Meet., 465 (2006) 